BROWN-HET-830

March 1992

\title{
SUPERCONDUCTING COSMIC STRINGS AND PRIMORDIAL MAGNETIC FIELDS
}

\author{
Robert H. Brandenberger ${ }^{1)}$, Anne-Christine Davis ${ }^{2)}$, \\ Andrew M. Matheson ${ }^{1), 3)}$ and Mark Trodden ${ }^{4}$ ) \\ 1) Physics Department, Brown University \\ Providence, RI 02912, U.S.A. \\ 2) Department of Applied Mathematics and \\ Theoretical Physics and Kings College \\ University of Cambridge \\ Cambridge CB3 9EW, U.K. \\ 3) 2 Soldiers Field Park \#803 \\ Harvard Business School \\ Boston, MA 02163, U.S.A. \\ 4) Churchill College \\ University of Cambridge \\ Cambridge, U.K.
}

\begin{abstract}
We consider grand unified theories with superconducting cosmic strings and which admit the mechanism for generating primordial magnetic fields recently discussed by Vachaspati. We show that these models are severely constrained by cosmological arguments. Quite generically, either stable springs or vortons will form. Provided the mass per unit length of the strings is sufficiently large, these stable configurations will overclose the Universe.
\end{abstract}




\section{Introduction}

The possibility [1] that some models incorporating cosmic strings [2] may, under certain conditions, act as superconducting loops and carry large currents (large meaning currents of the order of magnitude of the string mass scale, expressed in appropriate units), spawned a rash of speculation on the possible manifestations of "superconducting strings" (for a review see e.g. Ref. 3). Missing from the menagerie of bizarre cosmological exotica, however, was a mechanism to provide the requisite magnetic fields that would act as seeds for the string currents.

Recent work [4] has illuminated one way of generating primordial magnetic fields at very early times in the evolution of the universe. Indeed, it is possible that coherent magnetic fields ( $\underline{B}$ fields hereafter) could be generated in sub-horizon sized patches during a large class of grand unified transitions. An obvious consequence of producing these $\underline{B}$-fields would be to charge up any superconducting string loops, quite conceivably formed in the same transition that gives rise to the $\underline{B}$-fields.

It is the ultimate fate of these primordial loops of string that concerns us in this note. If no $\underline{B}$-fields are present, the loops will shrink and disappear. If $\underline{B}$-fields are present, and have a coherent component that extends over a region as large as the loop, the loop will develop a current as it collapses. The current will induce forces tending to resist the string tension of the loop [5], hence diminishing the rate of decay of the loop and prolonging its life. However, the current cannot exceed a maximal value $j_{\max } \sim e \sqrt{\mu}$ (where $\mu$ is the mass per unit length of the string), since if $j>j_{\text {max }}$ it becomes energetically favourable for the charge carriers to jump off the string, leading to strong electromagnetic radiation [6]. There are hence two possible ultimate fates for a string loop. If the current can increase to $j_{\max }$, then the loop will decay by emitting a burst of electromagnetic radiation. If, on the other hand, the current $j_{\text {spring }} \sim \sqrt{\mu}[5]$ for which the force induced by electromagnetism balances the tension is smaller than $j_{\max }$, then stable loops ("springs" [5]) result. The fate of the loop is a parameter-dependent question. In some models, stable springs will form, while in others they will not $[5,7]$.

There is a second and more generic class of stable string configurations: vortons [8 - 11]. These result from strings with a net charge and induced angular momentum. As 
discussed in [11], strings with net charge will be generically produced by intersections of current carrying strings. The charge acts to further stabilize the configurations against collapse. This provides a mechanism by which stable objects form even if $j_{\text {spring }}>j_{\max }$.

Our claim is that all cosmic string models that admit springs or vortons, and which lead to primordial $\underline{B}$-fields at temperatures above a critical temperature $T_{i}$ (to be determined below) are ruled out. We can eliminate these theories because, as Copeland et al. observed [5], stable springs produce an overdensity of matter in the same manner as do primordial magnetic monopoles [12]. A necessary caveat is that the mass per unit length of strings should be sufficiently large (This bound will be discussed at the end of the Letter). If the strings are to have some relevance for galaxy formation, their mass per unit length $\mu$ must be about $10^{21} \mathrm{kgm}^{-1}\left(\left(10^{16} \mathrm{GeV}\right)^{2}\right)$. In many particle physics models the strings could be much lighter, but such strings are, by and large, uninteresting for cosmology, and we exclude them from consideration.

The outline of this Letter is as follows. We first review Vachaspati's mechanism [4] for the generation of magnetic fields. Next, we study the increase of currents on a cosmic string loop and determine the mass density in cosmic springs at the time of nucleosynthesis. Demanding that this density not exceed the energy density in radiation leads to new cosmological bounds on particle physics models.

\section{Primordial Magnetic Fields}

To establish our result, we recap the work of [4]. Under very general conditions (that gauge fields acquiring mass during spontaneous symmetry breaking carry electric charge), a magnetic field $\underline{B}$, smooth on a length scale $r_{i}$, is expected to form during a phase transition at temperature $T_{i}$, with $B\left(T_{i}\right) \sim T_{i}^{2}$ and $r_{i} \sim \frac{1}{T_{i}}$. Using the temperature-time relationship appropriate to the radiation-dominated era (during which we take the transition to occur), this coherence length is a fraction $\frac{\lambda T_{i}}{M_{p l}}$ of the horizon size $t_{i}\left(T_{i}\right)$. As usual, $M_{p l}$ is the Planck mass and $\lambda^{2} \equiv \frac{4 \pi^{3}}{45} g^{*}$ is a constant determined by the effective number of spin degrees of freedom $g^{*}$ of the theory under consideration. Typically, $g^{*} \geq 200$ and $\lambda^{2} \geq 500$. 
Since the cosmological plasma is a very good conductor on the large scales we are interested in, we expect the lines of magnetic flux to be frozen into the plasma and the patches to retain a constant comoving size. Then, the magnetic field within a coherence length scales as

$$
B(t)=B\left(t_{i}\right)\left(\frac{t_{i}}{t}\right) .
$$

Equivalently, the flux through a single patch in which the magnetic field is uniform is conserved:

$$
\Phi_{\text {patch }}(t)=\pi r_{i}^{2} B\left(t_{i}\right) .
$$

On scales larger than the patch size, the flux has random orientation. Hence, the net flux through a horizon size loop enclosing $N^{2}$ patches each carrying a flux $\Phi_{\text {patch }}$ will be of the order

$$
\Phi_{n e t} \sim N \Phi_{\text {patch }} \sim\left(\frac{t}{t_{i}}\right)^{1 / 2} \frac{t_{i}}{r_{i}} \Phi_{\text {patch }}
$$

(There are $\left(t_{i} / r_{i}\right)^{2}$ patches at time $t_{i}$ enclosed by a horizon size loop).

\section{Springs}

The superconducting property of superconducting strings means that if a loop encloses a flux $\Phi_{0}$ at the time $t_{0}$ when the loop forms, it will generate a current to preserve the flux threading it at all later times. There are thus two contributions to the total flux threading a loop: $\Phi_{1}$ which is the flux induced by the coherent magnetic fields and $\Phi_{2}$ which is the flux induced by the current on the string. It is clear that

$$
\Phi_{2}(t)=2 \pi R(t) J(t)
$$

and so flux conservation:

$$
\frac{d}{d t}\left[\Phi_{1}+\Phi_{2}\right]=0
$$

yields our basic differential equation

$$
\frac{d J}{d t}=-\frac{J}{R} \frac{d R}{d t}-\frac{1}{2 \pi R} \frac{d \Phi_{1}}{d t}
$$

where $J$ is the current, $R$ is the size of the loop, and $\Phi_{1}$ is the flux through the loop (hereafter written as $\Phi$ ). In (3.3), the first term on the right hand side represents 
current build-up due to the collapse of the loop, while the second term represents current build-up due to the time-variation of the primordial B-field. From (3.2), we obtain

$$
\frac{d}{d t}(R J)=-\frac{1}{2 \pi} \frac{d}{d t}(\Phi)
$$

or

$$
J(t)=\frac{1}{2 \pi R(t)}[-\Phi(t)+\text { const }]
$$

Now, as $J(t)=0$ initially, and since $R\left(t_{0}\right)$ is finite,

$$
J(t)=\frac{1}{2 \pi R(t)}\left[\Phi\left(t_{0}\right)-\Phi(t)\right] .
$$

To interpret (3.6) we need to put in expressions for $R(t)$ (ignoring the back reaction of the current on $R(t)$ ) and $\Phi(t)$. The evolution of large loops is friction dominated immediately after the cosmic string producing phase transition. At later times, the decay of $R(t)$ is due to gravitational radiation [13]:

$$
R(t)=R\left(t_{0}\right)-\gamma G \mu\left(t-t_{0}\right)
$$

with $\gamma \sim 100$.

Consider now the flux through a loop of initial radius $R\left(t_{0}\right)$ formed at time $t_{0}$. From (2.3), the initial flux at time $t_{0}$ will be

$$
\Phi\left(t_{0}\right)=\Phi_{\text {patch }} \frac{R\left(t_{0}\right)}{r_{i}\left(t_{0} / t_{i}\right)^{1 / 2}}=\pi r_{i} B\left(t_{i}\right) R\left(t_{0}\right)\left(\frac{t_{i}}{t_{0}}\right)^{1 / 2}
$$

At later times $t>t_{0}$, the number of coherence regions inside the loop will drop and

$$
\Phi(t)=\Phi_{\text {patch }} \frac{R(t)}{r_{i}\left(t / t_{i}\right)^{1 / 2}}=\pi r_{i} B\left(t_{i}\right) R(t)\left(\frac{t_{i}}{t}\right)^{1 / 2} .
$$

Hence, from (3.6)

$$
J(t)=\frac{1}{2} r_{i} B\left(t_{i}\right) \frac{R\left(t_{0}\right)}{R(t)}\left(\frac{t_{i}}{t_{0}}\right)^{1 / 2}\left[1-\frac{R(t)}{R\left(t_{0}\right)}\left(\frac{t_{0}}{t}\right)^{1 / 2}\right] \equiv J_{0} \frac{R\left(t_{0}\right)}{R(t)}\left[1-\frac{R(t)}{R\left(t_{0}\right)}\left(\frac{t_{0}}{t}\right)^{1 / 2}\right]
$$


with

$$
J_{0}=\frac{1}{2} r_{i} B\left(t_{i}\right)\left(\frac{t_{i}}{t_{0}}\right)^{1 / 2}
$$

The time dependence of $J(t)$ can be inferred from (3.10). Since $R(t)$ decreases only very slowly, the current $J(t)$ rapidly rises to the value $J_{0}$ and remains approximately constant until (see (3.7)) the time

$$
t_{d}=(\gamma G \mu)^{-1} R\left(t_{0}\right)
$$

at which time the current once again increases sharply during the period when the loop radius decreases from the value $\gamma G \mu R\left(t_{0}\right)$ to a final value at which the loop becomes a spring, or when the maximal current is reached, whichever occurs first. During this final collapse phase, the product $R(t) J(t)$ is constant.

Using the values

$$
r_{i}=\alpha_{1} T_{i}^{-1} \text { and } B\left(t_{i}\right)=\alpha_{2} T_{i}^{2}
$$

for the initial coherence length and magnitude of the magnetic field (where $\alpha_{1}$ and $\alpha_{2}$ are constants which depend on the details of the phase transition), then in the case $t_{0}=t_{1}$ when the cosmic strings form in the same phase transition as the magnetic field, the current $J_{0}$ becomes

$$
J_{0}=\frac{1}{2} \alpha_{1} \alpha_{2} T_{i}=\alpha_{3} \sqrt{\mu}=x j_{\text {spring }}
$$

where $x$ is a combination of $\alpha_{1}, \alpha_{2}$ and the constants $j_{\text {spring }} / \sqrt{\mu}$ and $T_{i} / \sqrt{\mu}$. Thus, we conclude that for $t_{0}=t_{1}$ already the "initial" current $J_{0}$ can be of the right order of magnitude to give springs.

When $T_{0}=T_{2}<T_{1}$, where $T_{2}$ is the temperature of the cosmic string producing phase transition, the relation between $J_{0}$ and the spring current is unchanged since

$$
J_{0}=\frac{1}{2} \alpha_{1} \alpha_{2} T_{2}=\alpha_{3} \sqrt{\mu}=x j_{\text {spring }} .
$$

Consider now the case $x \ll 1$. In order for such a model with $j_{\text {spring }}<j_{\max }$ to be cosmologically safe, the current $J_{0}$ must be sufficiently small such that $J(t)$ 
remains smaller than $j_{\text {spring }}$ until $R(t)$ shrinks to a size comparable to the thickness $w \sim(\mu) \lambda-1 / 2$ of the string, independent of the temperature $T_{0}<T_{2}$ when the loops are split off from the network of infinite cosmic strings. A loop formed at temperature $T_{0}$ has a typical size $R_{i}=\alpha t_{0}\left(T_{0}\right)$, equal to

$$
R_{i}=\frac{\alpha}{\lambda} \frac{M_{p l}}{T_{0}^{2}}
$$

and develops a current

$$
J_{0}=x \frac{T_{0}}{T_{2}} j_{\text {spring }}
$$

due to the magnetic flux threading the loop. While collapsing to size $w$, the current increases by a factor

$$
f=\frac{R_{i}}{w}=\frac{\alpha M_{p l}}{\lambda T_{0}^{2} w} .
$$

For the current at this point not to exceed $j_{\text {spring }}$ we must have

$$
x \frac{T_{0}}{T_{2}} f=x \frac{\alpha}{\lambda} \frac{M_{p l} w^{-1}}{T_{2} T_{0}}<1 .
$$

At high temperatures immediately after the cosmic string producing phase transition, the motion of strings is dominated by the friction of the surrounding plasma. In the absence of primordial $\underline{B}$-fields, the loops formed would simply shrink and disappear. However, in the presence of primordial B-fields these loops can only shrink to a final radius

$$
R_{f}=x \frac{\alpha}{\lambda} \frac{M_{p l}}{T_{0}} \frac{1}{T_{2}}
$$

when $J=j_{\text {spring }}$, and then become springs. Our results therefore apply equally well to the friction-dominated case.

From (3.19), we see that independent of how small the constants $x$ and $\alpha / \lambda$ are (according to the recent cosmic string evolution simulations [14] $\alpha \sim 10^{-3}$ ), eventually $T_{0}$ will become sufficiently small such that the condition is violated, and hence springs will form. The presence of springs causes cosmological problems. They might 
overclose the Universe (see e.g. Ref. [15]). More specifically, successful nucleosynthesis requires that Universe is still radiation dominated at the time of nucleosynthesis. The only ways to satisfy this constraint are to make the strings sufficiently light, or to make sure that no $\underline{B}$-fields are produced above a certain temperature.

Taking $T_{0}$ to be $T_{2}$ or the temperature when the inequality (3.19) is marginal (whichever is lower), then the mass of the earliest spring, whose radius is given by $(3.20)$, is

$$
M_{\text {loop }}=2 \pi \mu R_{f} \sim 2 \pi \frac{\alpha}{\lambda} x \frac{M_{p l}}{T_{0}} \sigma
$$

where $\sigma=\sqrt{\mu}$ is the scale of symmetry breaking. If there is one loop of radius $R_{i}$ per horizon volume at temperature $T_{0}$ which forms a spring (it is such loops which dominate the total mass - see e.g. Ref. [16]), then there will be $\left(\frac{T_{0}}{T_{F}}\right)^{3}$ springs per horizon volume at a lower temperature $T_{F}$, and a total mass of springs inside the horizon of

$$
M_{t o t}=2 \pi \frac{\alpha}{\lambda} x \frac{M_{p l}}{T_{0}} \sigma\left(\frac{T_{0}}{T_{F}}\right)^{3}
$$

In particular, if the Universe is to be radiation-dominated at nucleosynthesis when $T_{F}=0.1 \mathrm{MeV}$, the energy in springs must be less than that in radiation. We therefore require that the ratio

$$
4 \pi \alpha x \frac{\sigma}{T_{F}}\left(\frac{T_{0}}{M_{p l}}\right)^{2}<<1
$$

If $x \approx 1$ and $T_{0}=T_{2}$, radiation-dominated nucleosynthesis implies that $T_{2}<<$ $10^{11} \mathrm{GeV}$ if $\underline{\mathrm{B}}$ - fields are present. For strings with potential to form galaxies, the temperature at which the strings are formed is $T_{2} \approx 10^{15} \mathrm{GeV}$. Our constraint (3.23) (this time evaluated with $T_{0} \neq T_{2}$ ) therefore implies no $\underline{B}$-fields may be produced in superconducting spring theories above $T_{i} \equiv T_{0} \sim 10^{10} \mathrm{GeV}$. For radiation-domination down to $T=1 \mathrm{eV}$, our limit is $T_{0} \leq 3 \times 10^{7} \mathrm{GeV}$. If, on the other hand, we assume $T_{0}=\sigma \neq 10^{15} \mathrm{GeV}$, then (3.23) yields the constraint $T_{0}<10^{12} \mathrm{GeV}$ for radiation domination to $T_{F}=0.1 \mathrm{MeV}$. 


\section{Vortons}

Let us now consider vortons, a class of stable field configurations more generic than springs, arising also if $j_{\text {spring }}>j_{\max }$. The essential difference between vortons and the springs considered above is that the vortons have charge and angular momentum [8 -10]. This feature leads to the vorton solutions being intrinsically more stable than the corresponding spring solutions and therefore posing a more serious cosmological problem in the type of theories considered above.

We consider as a simple example the bosonic $U(1) \times U(1)_{e m}$ model originally proposed by Witten [1]. The Lagrangian contains two scalar fields, $\hat{\phi}$ which gives rise to the strings and $\hat{\sigma}$ which condenses inside the string and induces the superconducting current.

Defining $\phi \equiv|\hat{\phi}|, \sigma \equiv|\hat{\sigma}|$, and $\theta \equiv \arg \hat{\sigma}$, there exist two currents on the vortex worldsheet, one which is conserved topologically and gives the string a current

$$
\tilde{j}_{a}=\epsilon_{a b} \partial^{b} \theta,
$$

and a second which is conserved by the equations of motion and gives the vortex an electric charge

$$
j_{a}=\sigma^{2}\left(\partial_{a} \theta-A_{a}\right)
$$

( $A_{a}$ being the electromagnetic potential) with respective charges

$$
N=2 \pi R n \quad Q=\oint d l \int_{X} \sigma^{2}\left(\omega-A_{0}\right) .
$$

Here, $Q$ is the electromagnetic charge, and $R$ is the average radius of the loop. Also $\omega \equiv \partial_{0} \theta$ and $\int_{X}$ is the integral over the string cross-section.

The presence of a non-zero $Q$ on the string causes the loop to have angular momentum, and in the case of chiral vortons for which $\omega=n$, we have

$$
\theta=\omega(t-l),
$$

where $l$ is the arc length around the loop. Since $\omega / n=1$ can be shown [8] to be an attractor, chiral vortons are expected to dominate, and hence we shall focus on them. 
For chiral vortons, the total electromagnetic charge on a loop is proportional to the topological charge $N$. It will prevent the loop from collapsing and there will be a finite stabilization radius $R_{F}$ which can be computed by minimizing the total static energy [10] given by:

$$
E=2 \pi R \mu+4 \pi \frac{N^{2}}{R} \Sigma
$$

where $\Sigma$ is an integral of $\sigma^{2}$ over the string cross section, to yield

$$
R_{F} \simeq \frac{N}{\sqrt{4 \pi} \sigma}
$$

Models with vortons can cause cosmological problems if $R_{F}$ is larger than the width $w \sim \sigma^{-1}$ of the string. In the other case, the loop will simply disappear in a burst of elementary quanta. A loop of initial radius $R_{0}$ will carry an average initial topological charge $N$ of

$$
N=\frac{R_{0}}{\xi\left(T_{0}\right)}
$$

where $\xi\left(T_{0}\right) \sim T_{0}^{-1}$ is the correlation length of the phase of $\hat{\phi}$ at the time of loop formation. Hence,

$$
R_{F}=\frac{\alpha}{\sqrt{4 \pi} \lambda} \frac{M_{p l}}{T_{0}} \frac{1}{\sigma}
$$

which, up to factors of order unity, agrees with the final radius (3.20) of cosmic springs.

Equation (4.8) leads to interesting consequences. If $R_{F}<\frac{1}{\sigma}$, then no stable vortons can form. With the values of $\alpha$ and $\lambda$ given before, this occurs for $T_{0}>$ $10^{14} \mathrm{GeV}$. In models with $\sigma>10^{14} \mathrm{GeV}$, vortons will hence not immediately form, but only once the temperature of the Universe drops below this value. If $\sigma<10^{14} \mathrm{GeV}$, vortons form immediately after the phase transition.

Since the stabilization radius of a vorton agrees with the final spring radius, the mass bounds are identicial to those discussed at the end of Section 3. We conclude that all theories with $\sigma>10^{10} \mathrm{GeV}$ admitting superconducting cosmic strings are ruled out; those with $j_{\max }>j_{\text {spring }}$ by springs, those with $j_{\max }<j_{\text {spring }}$ by vortons. 
In addition, the presence of charge on strings modifies the considerations of Section 3. There are now two contributions to the total current, that coming from the string current which we denote by $J_{c}$ and that coming from the charge (denoted by $J_{Q}$ ). The final spring radius $R_{f}$ is now determined by equality between total current and spring current. Hence, for given initial winding number and radius, $R_{F}$ is larger with charge than without. Hence, the mass bounds are stronger.

First, we determine for which $J_{Q}$ substantial changes to the results of Section 3 can be expected. This will be the case if for loops of radius $R_{F}$ (see (3.20)) the current $J_{Q}$ exceeds $j_{\text {spring }} \sim \sigma$. With $[10]$

$$
Q \sim e \frac{R_{i}}{\xi\left(T_{0}\right)}
$$

and

$$
J_{Q}(t) \sim \frac{\omega Q}{2 \pi}
$$

we see that for

$$
T_{0}<\frac{e}{4 \pi^{2}} \frac{\alpha}{\lambda} M_{p l} \sim 10^{13} \mathrm{GeV}
$$

there will be a substantial modification of the previous results. The new stabilization radius $\tilde{R_{F}}$ is determined by $J_{Q}=\sigma$ which gives

$$
\tilde{R}_{F}=\frac{e}{4 \pi^{2}}\left(\frac{\alpha}{\lambda}\right)^{2}\left(\frac{M_{p l}}{T_{0}}\right)^{2} \frac{1}{\sigma}
$$

To determine the new constraints which result when taking into account vortons, we redo the mass estimates of $(3.21-3.23)$ with $\tilde{R}_{F}$ instead of $R_{F}$. A simple calculation gives the following results: for $\sigma=10^{15} \mathrm{GeV}$, the temperature $T_{0}$ at which the magnetic field is created is bounded by

$$
T_{0}<2 \times 10^{6} \mathrm{GeV}
$$

(with $T_{F}=0.1 \mathrm{MeV}$ ). Alternatively, if we assume that $T_{0}=\sigma \neq 10^{15} \mathrm{GeV}$, the 
constraint becomes

$$
T_{0}<3 \times 10^{10} \mathrm{GeV}
$$

These bounds are several orders of magnitude stronger than the ones obtained when neglecting vortons.

\section{Conclusions}

To summarize, we have derived constraints on models admitting superconducting cosmic strings. We have assumed that at some temperature $T_{0}$, primordial magnetic fields are produced [4] during one of the phase transitions which breaks the original symmetry group down to the standard model. The constraints come from demanding that stable springs or vortons do not cause the Universe to become matter dominated before nucleosynthesis.

Focusing on cosmic string models with a scale of symmetry breaking $\sigma \sim 10^{15} \mathrm{GeV}$ (the value required in galaxy formation scenarios [17]), we distinguish the following cases. If the spring current $j_{\text {spring }}$ is larger than the maximal current $j_{\text {max }}$, then there are only stable vortons and the constraint on $T_{0}$ is $T_{0}<10^{10} \mathrm{GeV}$. For $j_{\text {spring }}<$ $j_{\max }$, both stable springs and vortons exist. Vortons dominate the mass density if $T_{0}<10^{13} \mathrm{GeV}$, springs are more important otherwise. In such models, the Universe is matter dominated at nucleosynthesis unless $T_{0}<2 \times 10^{6} \mathrm{GeV}$.

For cosmic strings of relevance for structure formation, the phase transition producing $\underline{B}$-fields cannot occur much above the electroweak scale. If such fields are produced at the grand unified scale, then the mass per unit length of the superconducting cosmic strings cannot be sufficiently large for the strings to be of relevance in structure formation scenarios.

In analyzing models with $j_{\text {spring }}>j_{\max }$, we have assumed that the charge on a loop remains constant while it is emitting electromagnetic radiation. In a perturbative analysis, this is obvious. However, nonperturbative processes may allow net charge decrease (e.g. by further loop fragmentation).

\section{Acknowledgements}


This work has been supported in part by DOE grant DE-AC02-76ER03130 Tasks A \& K, by an Alfred P. Sloan Fellowship to R.B., and by an NSF- SERC Collaborative Research Award NSF INT-9022895 and SERC GR/G37149. 


\section{REFERENCES}

1. E. Witten, Nuc. Phys. B249 (1985) 557.

2. T. W. B. Kibble, J. Phys A9 1387;

A. Vilenkin, Phys. Rep. 121263.

3. E. Copeland, Cosmic Strings and Superconducting Cosmic Strings, in Proc. of Second Erice Summer School on Dark Matter, Erice, May 1988 (World Scientific, Singapore, 1988).

4. T. Vachaspati, Phys. Lett. 265B (1991) 258.

5. E. Copeland, M. Hindmarsh and N. Turok, Phys. Rev. Lett. 58 (1987) 1910.

6. G. Field and A. Vilenkin, Nature 326 (1986) 944;

J. Ostriker, C. Thompson and E. Witten, Phys. Lett. 180B (1986) 231;

D. Spergel, T. Piran and J. Goodman, Nucl. Phys. B291 (1987) 847;

E. Copeland, D. Haws, M. Hindmarsh and N. Turok, Nucl. Phys. B306 (1988) 908.

7. A. Babul, T. Piran and D. Spergel, Phys. Lett. 202B (1988) 307;

C. Hill, H. Hodges and M. Turner, Phys. Rev. D37 (1988) 263;

D. Haws, M. Hindmarsh and N. Turok, Phys. Lett. 209B (1988) 255.

8. R. Davis and E.P.S. Shellard, Phys. Lett. 207B (1988) 404;

R. Davis and E.P.S. Shellard, Phys. Lett. 209B (1988) 485.

9. R. Davis, Phys. Rev. D38 (1988) 3722.

10. R. Davis and E.P.S. Shellard, Nucl. Phys. B323 (1989) 209.

11. T.J. Allen, Phys. Lett. 231B (1989) 429;

T.J. Allen, Phys. Lett. 250B (1990) 29.

12. M. Khlopov and Ya. B. Zel'dovich, Phys. Lett. 79B (1978) 239;

J. Preskill, Phys. Rev. Lett. 43 (1979) 1365.

13. N. Turok, Nucl. Phys. B242 (1984) 520;

T. Vachaspati and A. Vilenkin, Phys. Rev. D31 (1985) 3052;

C. Burden, Phys. Lett. 164B (1985) 277. 
14. A. Albrecht and N. Turok, Phys. Rev. D40 (1989) 973;

D. Bennett and F. Bouchet, Phys. Rev. Lett. 60 (1988) 257;

B. Allen and E.P.S. Shellard, Phys. Rev. Lett. 64 (1990) 119.

15. A.-C. Davis and R. Brandenberger, Cosmological Limits on Stable Particle Production at High Energy, Brown preprint BROWN-HET-828 (1991) Phys. Lett. B (in press).

16. T. Vachaspati and A. Vilenkin, Phys. Rev. D30 (1984) 2036;

D. Mitchell and N. Turok, Phys. Rev. Lett. 58 (1987) 1577.

17. N. Turok and R. Brandenberger, Phys. Rev. D33 (1986) 2175;

A. Stebbins, Ap. J. (Lett.) 303 (1986) L21;

H. Sato, Prog. Theor. Phys. 75 (1986) 1342. 